\title{
Cisplatin-Digoxigenin mRNA Labeling for Nonradioactive Detection of mRNA Hybridized onto Nucleic Acid cDNA Arrays
}

\author{
T. Hoevel, H. Holz ${ }^{1}$ and M. Kubbies \\ Roche Pharmaceutical Research, and ${ }^{1}$ Roche Molecular Diagnostics, Penzberg, Germany
}

BioTechniques 27:1064-1067 (November 1999)

\begin{abstract}
We optimized a novel nonradioactive hybridization technique using a cis-platin coupled digoxigenin derivative for direct labeling of mRNA. This new mRNA reporter molecule was applied to cDNA membrane arrays to simultaneously identify expression of hundreds of genes. The sensitivity of this nonradioactive mRNA hybridization technique was comparable to radioactive cDNA labeling on housekeeping gene expression but even superior on the detection limit of the expression of low abundant genes using $\mathrm{mRNA}$ isolated from human diploid fibroblasts. Additional advantages are faster readout and decreased total working times because of luminescence technology and avoidance of radioactivity. Finally, no potential artifactual reverse transcription step is necessary because of direct labeling of mRNA used for hybridization on nucleic acid arrays.
\end{abstract}

\section{INTRODUCTION}

Nucleic acid arrays are powerful tools used to compare differential gene expression of genetic or physiologically different cell types $(2,4,5,6,8)$. Northern analysis, ribonuclease protection assay or coupled reverse transcription (RT) and subsequent PCR focus on the expression analysis of one or more genes. With the more recent developments of nucleic acid arrays $(11,12)$ and high-density oligonucleotide arrays $(3,10)$, the simultaneous evaluation of the expression of several hundreds to thousands of genes became reality. Nucleic acid arrays consist of different cDNAs spotted onto array membranes. Radioactive cDNA generated from mRNA is hy- bridized onto the membrane and subsequently visualized by X-ray film exposition (9).

To identify new genes relevant for DNA repair, cell cycle disturbances and carcinogenesis, a comparison of gene expression of normal lung and Fanconi anemia fibroblasts was performed (13) by applying the promising technology of multiple cDNA arrays. However, because of unspecific hybridization background combined with lack of sensitivity on wellexpressed non-housekeeping or low-abundance genes using the radioactive ${ }^{32} \mathrm{P}$ cDNA labeling technique, we applied and optimized successfully a nonradioactive hybridization technique based on direct mRNA labeling and luminescence analysis.

\section{MATERIALS AND METHODS}

\section{RNA Isolation}

Normal diploid human lung fibroblasts were cultured to subconfluency in MEM medium supplemented with $10 \%$ fetal calf serum (13). To increase the purity of the mRNA, two different extraction steps were applied sequentially. The cell samples were lysed under denaturing conditions with isothiocyanate and $\beta$-mercaptoethanol, and total RNA was then isolated using a silica gel-based membrane for RNA binding (QIAGEN, Valencia, CA, USA). Total RNA was hybridized to biotin-labeled oligo(dT) ${ }_{20}$ probes, and mRNA was isolated using streptavidin-coupled magnetic beads (Roche Molecular Biochemicals, Mannheim, Germany) followed by a purifica- 
tion step with phenol/chloroform and ethanol (1). Aliquots of identical samples were stored at $-80^{\circ} \mathrm{C}$ and used in parallel for nonradioactive and radioactive probe labeling.

\section{Direct Labeling of mRNA}

For non-radioactive mRNA labeling, we used a novel cisplatin-coupled digoxigenin (DIG) compound (DIG-ChemLink; Roche Molecular Biochemicals). This compound consists of a DIG moiety, which is bound by an aliphatic linker to a cisplatin complex. Two of the four binding sites of the cisplatin complex consist of a diaminoethane ligand. The third binding site is covalently bound to the linker/DIG molecule, and the fourth binding site is a cleavable nitrate ligand. Incubation in an aqueous solution with nucleic acid templates (DNA or RNA) cleaves the nitrate and a complex is formed between the cisplatin and the $\mathrm{N}_{7}$ position of $\mathrm{G}$ and $\mathrm{A}$ bases. The coordinative complex is stable and resistant to nucleic acid denaturation. This cisplatin-DIG compound $(2 \mu \mathrm{L})$ was incubated at $85^{\circ} \mathrm{C}$ for $30 \mathrm{~min}$ with different amounts of mRNA $(0.7-2.0 \mu \mathrm{g})$, and the reaction was terminated by the

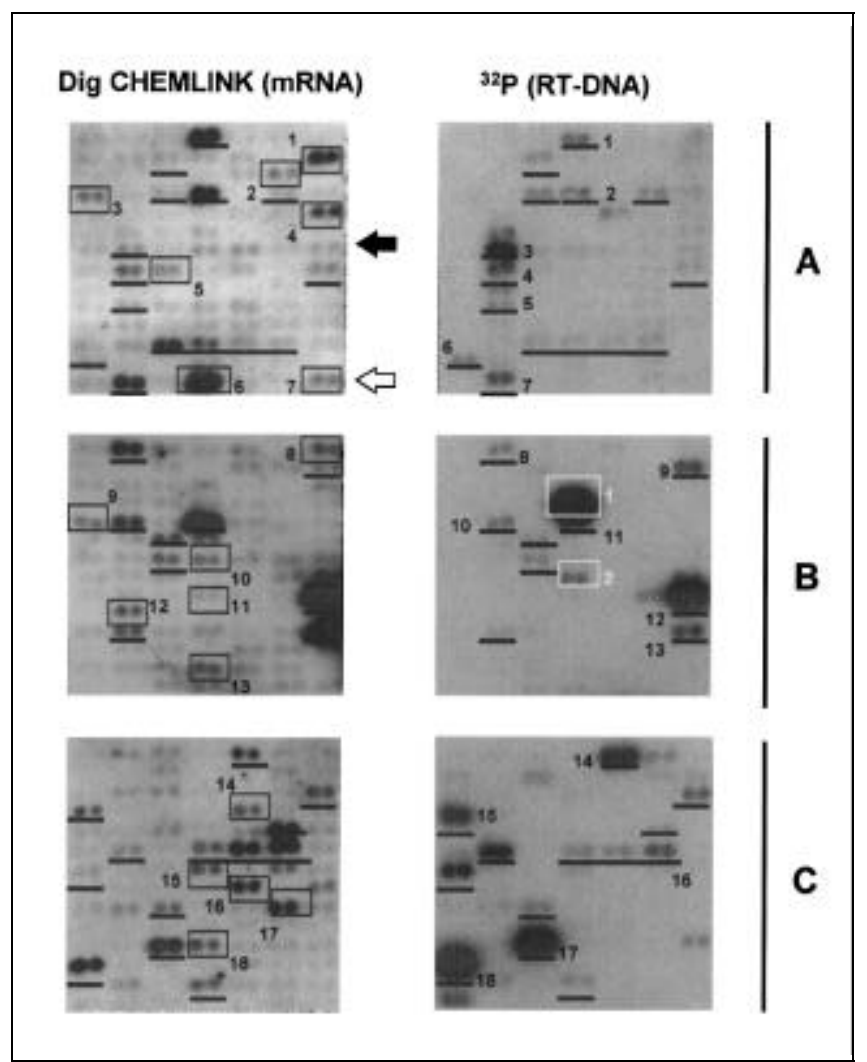

Figure 1. Comparison of nonradioactive and radioactive detection of hybridization signals of mRNA and RT DNA on Atlas human cDNA expression arrays. Biological target specificity of the Atlas human cDNA expression arrays: (A) apoptosis, DNA synthesis/repair/recombination-related genes, (B) growth factors/cytokines/chemokines/interleukins/interferons/hormones and (C) transcription factors/DNA binding proteins. Bars 1-18 indicate expressed genes detected significantly by both nonradioactive and radioactive detection technology (Table 1). Unnumbered bars indicate genes that enhance the similarity pattern of both arrays. Boxes 1-18 indicate expressed genes detected only by the nonradioactive DIG-ChemLink technique (Table 1). The white boxes display genes with significantly increased spot intensity or that are detected only by the radioactive labeling technique. The black and white arrows indicate twin spot rows used for image intensity profile analysis as shown in Figure 2. addition of a stop solution $(7,14)$. For the radioactive detection of the DNA-DNA hybrids, we performed the RT step of the mRNA and subsequent ${ }^{32} \mathrm{P}$ labeling with a specific probe activity of $5-10 \times 10^{6} \mathrm{cpm}$ according to the manufacturer's guidelines (CLONTECH Laboratories, Palo Alto, CA, USA).

\section{Hybridization and Detection}

We compared nonradioactive and radioactive detection methods of gene expression in fibroblasts with the Atlas ${ }^{\mathrm{TM}}$ human cDNA expression array technology (CLONTECH Laboratories). To suppress unspecific hybridization, we performed a prehybridization step of the arrays for $1 \mathrm{~h}$ at $50^{\circ} \mathrm{C}$ using a formamide-free hybridization solution containing fish sperm DNA (Roche Molecular Biochemicals). We performed hybridization overnight at $50^{\circ} \mathrm{C}$ in roller flasks using $8 \mathrm{~mL}$ of hybridization solution DIG Easy Hyb supplemented with $5 \mu \mathrm{g}$ of COT-1 DNA (both from Roche Molecular Biochemicals) and $0.7-1.4 \mu \mathrm{g}$ of $\mathrm{mRNA}$ probe, both denatured for $5 \mathrm{~min}$ at $95^{\circ} \mathrm{C}$. Because RNA-DNA hybrids are less stable than RNARNA hybrids, the stringency washes were performed at $50^{\circ} \mathrm{C}$, and DIG detection was performed according to the manufacturer's instructions. The radioactive labels on the cDNA membrane arrays were visualized on X-ray film (Eastman Kodak, Rochester, NY, USA; 7 days exposure). The chemiluminescence signals generated by CDP-Star ${ }^{\mathrm{TM}}$ substrate con-version were detected by a chemiluminescence detection film (5-30 min exposure) and scanned with the Lumi-Imager ${ }^{\mathrm{TM}}$ (both from Roche Molecular Biochemicals). The exposition times for both recording systems were chosen to obtain identical spot intensities for the genomic controls and housekeeping genes. From both film types, Tagged Image File Format (TIFF), files were generated, and the spot intensities were analyzed and evaluated by line scan profiling after nearest neighbor background subtraction using the Model 570 Quantimet $^{\circledR}$ Image Analysis System (Leica, Bensheim, Germany).

\section{RESULTS}

Figure 1 shows the direct comparison of selected Atlas cDNA array quadrants using nonradioactive labeling of mRNA with the cisplatin-DIG derivative (DIG-ChemLink; Figure 1, left row) and radioactive labeling of reverse transcription (RT)-DNA with ${ }^{32} \mathrm{P}$ (right row). The similarity of the dot pattern is striking, and the bars mark major identities. Within these three quadrants, the gene expression of 37 genes could be detected visually with the radioactive labeling technique as well as with the DIG-ChemLink technology (Figure 1 , all bars, left and right rows). The right row of Table 1 summarizes a selected number of well-defined genes. Besides the slightly improved signal-to-noise ratio using the cisplatindigoxigenin labeling technique, we identified significant differences in signal intensity for a variety of genes using the DIG-ChemLink technology compared to the RT-DNA ${ }^{32} \mathrm{P}$ technique. On one hand, on the DIG-ChemLink mRNA arrays, the genes marked by the bars $1,2,7,8,10,13$ and 16, for example, display a significant increased spot intensity compared to their ${ }^{32} \mathrm{P}$ RT-DNA counterparts. On the other hand, on the ${ }^{32} \mathrm{P}$ RT-DNA arrays, the genes marked by the bars 3, 9, 14, 17 and 18, for example, exhibit a manifold higher spot intensity (see Figure 1, bar labels in right row).

This slightly increased signal-to-noise ratio using the DIG- 
Table 1. Comparison of Gene Expression Detected by the DIG-ChemLink Nonradioactive mRNA Cisplatin-DIG Hybridization Technology and RTDNA ${ }^{32}$ P Radioactive Labeling Technique

\begin{tabular}{|c|c|}
\hline Nonradioactive & Nonradioactive and radioactive \\
\hline \multicolumn{2}{|c|}{ panel A } \\
\hline SOD-1 (box 1) & TRADD (TNFR-1 associated protein) (bar 1) \\
\hline ATP-dependent DNA helicase II (box 2) & NIP-3 (bar 2) \\
\hline Mdm-2 (box 3) & nucleoside diphosphate kinase B (bar 3) \\
\hline UV excision repair protein RAD23 (box 4) & BAX beta (bar 4) \\
\hline Ionizing radiation resistance protein (box 5) & clusterin (bar 5) \\
\hline Apoptotic cystein protease MCH4 (box 6) & TNR-receptor 55 kD (bar 6) \\
\hline DNAse X (box 7) & GST microsomal (bar 7) \\
\hline \multicolumn{2}{|c|}{ panel B } \\
\hline Placental ribonuclease inhibitor (box 8) & hepatom-derived growth factor (bar 8) \\
\hline TGF $\beta-1$ (box 9) & inhibin $\beta$ (bar 9) \\
\hline Endothelial-monocyte act. factor polypept.II (box 10) & glucose-6-phosphate isomerase (bar 10) \\
\hline INFy induced protein (box 11) & connective tissue growth factor (bar 11) \\
\hline Rantes protein (box 12) & IGF-BP3 (bar 12) \\
\hline Leukemia inhibitory factor (box 13) & follistatin-related protein (bar 13) \\
\hline \multicolumn{2}{|c|}{ panel C } \\
\hline Tristetraproline (zinc-finger transc. regulat.) (box 14) & Y box binding protein-1 (bar 14) \\
\hline Transcription factor ETR 101 (box 15) & DNA binding protein inhib. factor ID-3 (bar 15) \\
\hline DNA binding protein SATB1 (box 16) & p21 (bar 16; rightmost double spots) \\
\hline Homeobox protein HOX-A1 (box 17) & guanine nucleotide binding protein G-S (bar 17) \\
\hline Transcription initiation factor IIB (box 18) & $60 \mathrm{~S}$ ribosomal protein L6 (bar 14) \\
\hline
\end{tabular}

ChemLink technique cannot account solely for the increased detection sensitivity of gene expression. Numerous housekeeping and low abundant genes expressed in human diploid lung fibroblasts were detected using only the nonradioactive technique and not the ${ }^{32} \mathrm{P}$ RT-DNA procedure. These twin spots are marked by black boxes in the left row of Figure 1 (see also Table 1, left row). Within these rows are relevant housekeeping genes like SOD1 or ATP-dependent DNA-helicase II in the exponential growing fibroblast cell culture, or genes like the growth factors TGF $\beta-1$ or the IL- 8 family member rantes. So far, only two genes have been identified that have spot intensities significantly higher using the ${ }^{32} \mathrm{P}$ labeling technique: thymosin beta-10 and VEGF-related-protein (Figure 1, right row, white boxes 1 and 2).

To evaluate the performance of the nonradioactive and radioactive labeling and detection methods independent of the gene expression, the grey values of the genomic DNA twin spots of the Atlas cDNA arrays were quantitated by image analysis. The grey value of hybridized genomic twin spots with the radioactive ${ }^{32} \mathrm{P}$ labeling technique was $94.3 \pm 5.6$ in comparison with $134.4 \pm 21.8$ with the cisplatin-DIG labeling technique. These results are not indicative of a major difference of the detection sensitivity. However, the nonradioactive DIG-ChemLink technique is consistently superior to the ${ }^{32} \mathrm{P}$ labeling technology in its signal-to-noise ratio.

Figure 2 shows that the peak profiles from image line scans correspond to the expected expression pattern in fibroblasts. Using our technology for analysis and data evaluation of gene expression in fibroblasts, a peak grey value of 10 or more spots must be considered as biologically relevant expression. This becomes evident, for example, by the detection of low-level expression of the p53 and CD40L as well as the $\mathrm{mdm}^{2}$ in human lung fibroblasts when applying the nonradioactive cisplatin-DIG method (Figure 2, panels A and B, twin spots a; Table 1). The expression of these genes could not be detected by the conventional RT-DNA ${ }^{32} \mathrm{P}$ labeling technique as evident from Table 1 .

\section{DISCUSSION}

In this report, we describe for the first time a novel technique for direct, nonradioactive labeling of mRNA (7) and its application for cDNA array hybridization. The optimized method takes advantage of a cisplatin-DIG derivative for direct mRNA labeling and subsequent hybridization onto cDNA expression arrays. Significant technical advantages are the avoidance of radioactivity and faster signal readout within a few minutes because of luminescence technology. In addition, the DIG-ChemLink technique labels mRNA down to a sensitivity of $0.1 \mathrm{pg}$ per spot (data not shown) and shows slightly improved sensitivity of detecting hybridized spots compared to ${ }^{32} \mathrm{P}$ labeling. One explanation for this finding could be the increased stability of RNA/DNA over DNA/DNA hybrids. However, the purity of the mRNA is the major bottleneck for obtaining optimal and reproducible 
hybridization signals for the DIG-ChemLink and the ${ }^{32} \mathrm{P}$ labeling technique. A decreased purity of mRNA might result in low coupling efficiency and increasing background, which leads to insufficient signal-to-noise ratios.

For a series of genes, we also identified significantly different spot intensities on the cDNA expression arrays when comparing the nonradioactive $\mathrm{mRNA}$ and the radioactive RTDNA labeling and hybridization technique. Two effects could account for this finding. First, the RT step necessary for ${ }^{32} \mathrm{P}$ labeling of cDNA might introduce a significant bias of the spot intensities. There is an individual, optimal temperature for the RT-PCR step of mRNA species. However, the cDNA for array hybridization is generated at a single temperature. Second, the bp composition of the mRNA might alter the labeling efficiency with the DIG-ChemLink compound.

We suggest that the artificial RT step used for the generation of the radioactive labeled probe might be the major cause of the deviation of the expression pattern observed on several genes (Figure 1). This is supported by the fact that the majority of the genes were not detected by the ${ }^{32} \mathrm{P}$ labeling technique using the RT step (Table 1). In addition, we do not expect the variation of the $A / G$ content of mRNAs to be different enough to explain a decrease of a factor of 2-4 in labeling intensity as shown in Figure 1. The quantitative aberrations observed might potentially limit the interpretation of the biological relevance of spot quantification and deserves further evaluation.

In summary, we have shown the usefulness of the novel

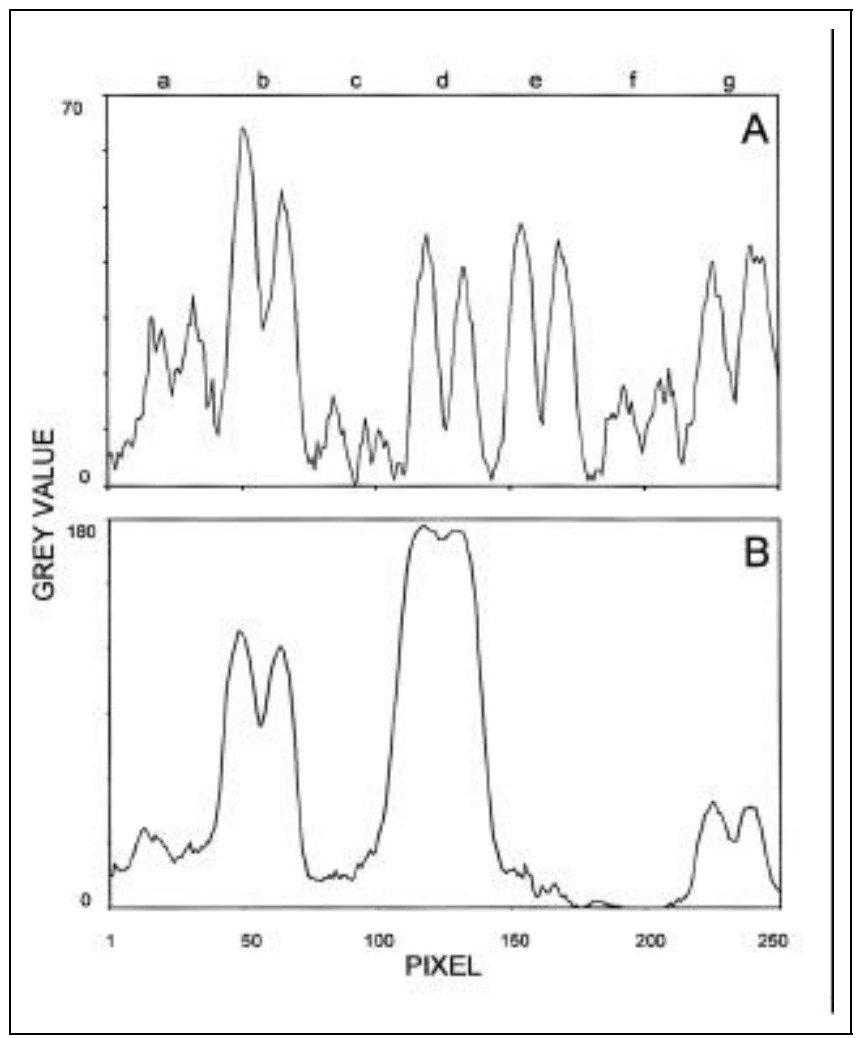

Figure 2. Signal intensity profiles of housekeeping and low abundant genes applying nonradioactive mRNA hybridization. Panel A and B correspond to intensity profiles from Figure 1 indicated by the black and white arrow, respectively. Double peaks correspond to twin spots of identical genes. Panel A: p53 (a), nucleoside diphosphate kinase (b), GST theta-1 (c), ICH-2 protease (d), BCL-W (e), replication factor C (f) and GADD 153 (g). Panel B: CD40 L (a), microsomal GST (b), lymphotoxin beta (c), apoptotic cystein protease MCH4 (d), RAD (e), ERCC2 (f) and DNase X (g). nonradioactive DIG-ChemLink mRNA labeling technique. However, because of the direct mRNA hybridization without any preceding RT amplification step, larger quantities of mRNA are necessary, which potentially limits the application at present to the field of in vitro cell models or larger tumor pieces. Besides the identification of the novel gene functions in our cell model using the nonradioactive mRNA labeling cDNA array technology (13), further studies are in progress in parallel to identify the biochemical parameters relevant for the bias of the detection of gene expression.

\section{REFERENCES}

1.Ausubel, F.M., R. Brent, R.E. Kingston, D.D. Moore, J.G. Seidman, J.A. Smith and K. Struhl. 1998. Current Protocols in Molecular Biology. Greene Publishing Associates and John Wiley \& Sons. New York.

2.Brown, P.O. and D. Botstein. 1999. Exploring the new world of the genome with DNA microarrays. Nat. Genet. 21:33-37.

3.Chee, M., R. Yang, E. Hubell, A. Berno, X.C. Huang, D. Stern, J. Winkler, D.J. Lockhart et al. 1996. Accessing genetic information with highdensity DNA arrays. Science 274:610-614.

4.DeRisi, J., L. Penland, O.O. Brown, M.L. Bittner, P.S. Meltzer, M. Ray, Y. Chen, Y.A. Su et al. 1996. Use of a cDNA microarray to analyse gene expression patterns in human cancer. Nat. Genet. 14:457-460.

5.Duggan, D.J., M. Bittner, Y. Chen, P. Meltzer and J.M. Trent. 1999. Expression profiling using cDNA microarrays. Nat. Genet. 21:10-14.

6.Heller, R.A., M. Schena, A. Chai, D. Shalon, T. Dedilion, J. Gilmore, D.E. Woolley et al. 1997. Discovery and analysis of inflammatory disease-related genes using cDNA microarrays. Proc. Natl. Acad. Sci. USA 94:2150-2155.

7.Holz, H., C. Goldstein, R. Rein and H.J. Hoeltke. 1997. DIG-ChemLink labeling and Detection Kit. Biochemica 3:26-27.

8.Lennon, G.G. and H. Lehrach. 1991. Hybridization analyses of arrayed cDNA libraries. Trends Genet. 7:314-317.

9.Lipshutz, R.J., S.P.A. Fodor, T.R. Gingeras and D.J. Lockhart. 1999. High density synthetic oligonucleotide arrays. Nat. Genet. 21:20-24.

10.Lockhart, D.J., H. Dong, M.C. Byrne, M.T. Follettie Gallo, M.S. Chee, M. Mittmann, C. Wang, M. Kobayashi et al. 1996. Expression monitoring by hybridization to high-density oligonucleotide arrays. Nat. Biotechnol. 14:1649.

11.Nguyen, C., D. Rocha, S. Granjeaud, M. Baldit, K. Bernard, P. Naquet and R.B. Jordan. 1995. Differential gene expression in the murine thymus assayed by quantitative hybridization of arrayed cDNA clones. Genomics 1:207-216.

12.Pietu, G., O. Alibert, V. Guichard, B. Lamy, F. Bois, E. Leroy, R. Mariage-Sampson, R. Houlgatte et al. 1996. Novel gene transcripts preferentially expressed in human muscles revealed by quantitative hybridization of a high density cDNA array. Genome Res. 6:492-503.

13.Planitzer, A.S., A.W. Machl, M. Rueckels and M. Kubbies. 1998. Identification of a novel c-DNA overexpressed in Fanconi's anemia fibroblasts partially homologous to a putative L-3-phosphoserine-phosphatase. Gene 210:297-306.

14.Van Belkum, A., E. Linkels, T. Jelsma, T.M. van den Berg and W. Quint. 1994. Non-isotopic labeling of DNA by newly developed haptencontaining platinum compounds. BioTechniques 16:148-154.

Address correspondence to Thorsten Hoevel, Roche Pharmaceutical Research, Dept. Cell Analytics, Nonnenwald 2, 82377 Penzberg, Germany. Internet: thorsten.hoevel@ roche.com 